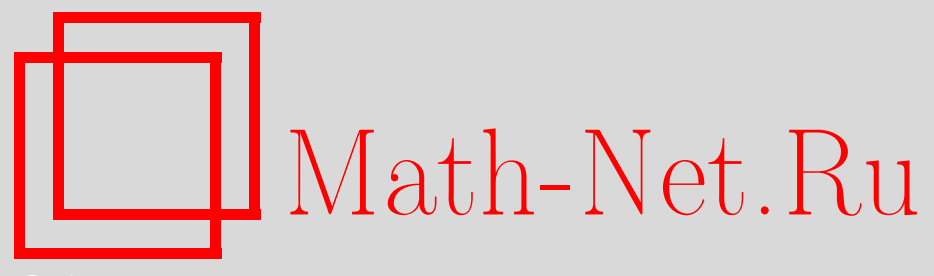

Э. Бак, Ю. В. Муранов, Нормальные инварианты пар многообразий и сигнатурные отображения, Матем. сб., 2006, том 197, номер 6, 3-24

DOI: https://doi.org/10.4213/sm1568

Использование Общероссийского математического портала Math-Net.Ru подразумевает, что вы прочитали и согласны с пользовательским соглашением http://www . mathnet.ru/rus/agreement

Параметры загрузки:

IP : 54.84 .234 .179

26 апреля 2023 г., 13:07:02

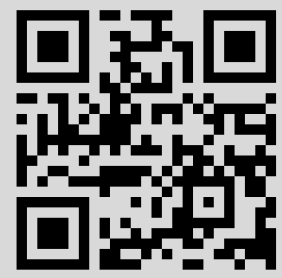




\author{
А. Бак, Ю. В. Муранов
}

\title{
Нормальные инварианты пар многообразий и сигнатурные отображения
}

\begin{abstract}
В статье строится структурное множество пары многообразий, описываются его нормальные инварианты и строятся диаграммы и косы точных последовательностей, связывающие введенное структурное множество и его нормальные инварианты со структурными множествами и нормальными инвариантами многообразия и подмногообразия.

Библиография: 15 названий.
\end{abstract}

\section{§1. Введение}

Пусть $X^{n}$ - связное замкнутое $n$-мерное топологическое многообразие с фундаментальной группой $\pi_{1}=\pi_{1}(X)$ и характером ориентации $w: \pi_{1} \rightarrow\{ \pm 1\}$. Фундаментальная проблема геометрической топологии состоит в описании всех возможных замкнутых $n$-мерных топологических многообразий, которые гомотопически (просто гомотопически) эквивалентны $X$.

Пусть $f: M \rightarrow X$ - сохраняющая ориентацию простая гомотопическая эквивалентность, где $M$ - связное замкнутое $n$-многообразие в категории ТОР, топологических многообразий. Два таких отображения $f_{i}: M_{i} \rightarrow X, i=1,2$, называются эквивалентными, если существует $s$-кобордизм $W$ между ними вместе с отображением $W$ в $X$, продолжающим отображения $f_{i}, i=1,2[1$; c. 542]. Множество классов эквивалентности образует структурное множество $\mathscr{S}_{n}^{s}(X)$ и входит в точную последовательность теории перестроек (см., например, [2; с. 278] и [1; с. 559])

$$
\cdots \longrightarrow[\Sigma X, G / \mathrm{TOP}] \stackrel{\sigma_{n+1}}{\longrightarrow} L_{n+1}\left(\pi_{1}\right) \longrightarrow \mathscr{S}_{n}^{s}(X) \longrightarrow[X, G / \mathrm{TOP}] \stackrel{\sigma_{n}}{\longrightarrow} L_{n}\left(\pi_{1}\right) .
$$

Элементы множества $[X, G / \mathrm{TOP}]$ называются нормалъными инвариантами. Для описания $\mathscr{S}_{n}^{s}(X)$ мы должны знать множество нормальных инвариантов, группы препятствий к перестройкам $L_{n}\left(\pi_{1}\right)=L_{n}^{s}\left(\pi_{1}\right)$ и сигнатурное отображение $\sigma$. Имеется значительный прогресс в вычислении $L$-групп (см. [3]-[6]), и получено много результатов о нормальных инвариантах и сигнатурных отображениях (см., например, [7]-[9]), но пока полученное описание $\mathscr{S}_{n}^{s}(X)$ остается в общем неудовлетворительным. Одной из значительных причин этого является трудность анализа сигнатурных отображений. Цель настоящей работы -

Работа первого автора поддержана фондом INTAS (грант № 00-566). Работа второго автора поддержана Российским фондом фундаментальных исследований (грант №05-0100993) и ДААД-программой для визита в Университет г. Биелефельда, Германия.

(C) А. БАК, Ю. В. Муранов, 2006 
получить информацию о сигнатурных отображениях, исследуя их отношение к подмногообразиям $X$.

Пусть $Y \subset X-$ подмногообразие коразмерности $q$ в $X$. Простая гомотопическая эквивалентность $f: M \rightarrow X$ расщепляется вдоль подмногообразия $Y$, если по определению она гомотопически эквивалентна трансверсальному к $Y$ отображению $g$ такому, что для $N=g^{-1}(Y)$ ограничения

$$
\left.g\right|_{N}: N \rightarrow Y,\left.\quad g\right|_{(M \backslash N)}: M \backslash N \rightarrow X \backslash Y
$$

являются простыми гомотопическими эквивалентностями. По определению $M \backslash N$ (аналогично $X \backslash Y$ ) является замыканием дополнения трубчатой окрестности $N$ (см. [9; с. 118]). Согласно [1; $\S 7.2]$ существует группа $L S_{n-q}(F)$ препятствий к расщеплению, которая зависит только от $n-q(\bmod 4)$ и универсальноотталкивающего квадрата

$$
F=\left(\begin{array}{ccc}
\pi_{1}(\partial U) & \longrightarrow & \pi_{1}(X \backslash Y) \\
\downarrow & & \downarrow \\
\pi_{1}(U) \longrightarrow & \pi_{1}(X)
\end{array}\right)
$$

фундаментальных групп с ориентацией, где $\partial U$ - граница трубчатой окрестности $U$ для $Y$ в $X$. Если $f: M \rightarrow X$ является нормальным отображением, то согласно $[1 ; \S 7.2]$ существует группа $L P_{n-q}(F)$ препятствий к перестройкам для пар многообразий $(X, Y)$, которая также зависит от $n-q(\bmod 4)$ и квадрата $F$. Группы $L S_{*}(F)$ и $L P_{*}(F)$ тесно связаны с группами препятствий к перестройкам $L_{*}\left(\pi_{1}(X)\right)$ и $L_{*}\left(\pi_{1}(Y)\right)$ и с классической точной последовательностью теории перестроек посредством коммутативной диаграммы

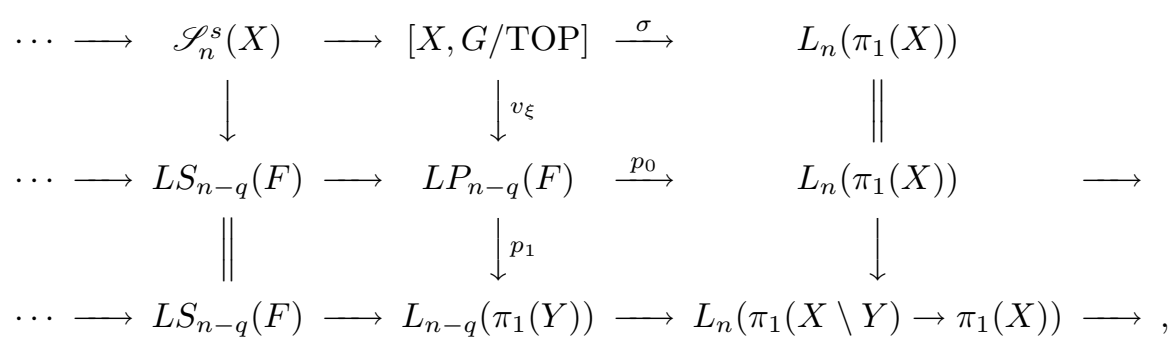

в которой все строки точны (см. [1; §7.2]) и [9; с. 136]).

В диаграмме отображения $p_{i}$ являются забывающими отображениями, а отображение $v_{\xi}$ определено естественным образом так, что композиция $p_{0} v_{\xi}$ является отображением $\sigma$ в классической точной последовательности теории перестроек. Необходимо отметить, что отображение $p_{1} v_{\xi}$ в диаграмме $(2)$ имеет естественное геометрическое описание. Любое нормальное отображение $h \in$ $[X, G / \mathrm{TOP}]$ дает посредством ограничения на подмногообразие $Y$ нормальное отображение из $[Y, G / \mathrm{TOP}]$. Препятствие к перестройке этого ограниченного отображения есть в точности $p_{1} v_{\xi}(h) \in L_{n-q}\left(\pi_{1}(Y)\right)$.

В [1; с. 571] А. А. Раницкий ввел множество $\mathscr{S}_{n}(X, Y, \xi) s$-триангуляций пары многообразий $(X, Y)$, где $\xi$ обозначает нормальное расслоение $Y$ в $X$. Это 
множество состоит из классов конкордантности $Y$-расщепленных отображений $f:(M, N) \rightarrow(X, Y)$, где $N=f^{-1}(Y)$, и входит в точную последовательность

$$
\cdots \longrightarrow \mathscr{S}_{n}(X, Y, \xi) \longrightarrow[X, G / \mathrm{TOP}] \stackrel{v_{\xi}}{\longrightarrow} L P_{n-q}(F) \longrightarrow \cdots .
$$

Эта последовательность продолжается вправо и влево, и множество $\mathscr{S}_{n}(X, Y, \xi)$ имеет групповую структуру. В [1; предложение 7.2.6] имеется описание свойств групп $\mathscr{S}_{n}(X, Y, \xi)$, включая диаграммы точных последовательностей, которые связывают между собой точные последовательности теории перестроек для многообразий $X$ и $Y$.

В настоящей работе мы вводим структурное множество $\mathscr{N}_{*}(X, Y)$ и показываем, что оно входит в точную последовательность

$$
\cdots \longrightarrow L_{n-q+1}\left(\pi_{1}(Y)\right) \longrightarrow \mathscr{N} \mathscr{S}_{n}(X, Y) \longrightarrow[X, G / \mathrm{TOP}] \stackrel{p_{1} v_{\xi}}{\longrightarrow} L_{n-q}\left(\pi_{1}(Y)\right)
$$

где, как обычно, точность в $L_{n-q+1}\left(\pi_{1}(Y)\right)$ и в $\mathscr{N} \mathscr{S}_{n}(X, Y)$ определяется в терминах действия группы $L_{n-q+1}\left(\pi_{1}(Y)\right)$ на $\mathscr{N}_{n}(X, Y)$ (теорема 1$)$. Определение $\mathscr{N}_{*}(X, Y)$ является естественным прямым обобщением (в котором $Y$ соответствует $\partial X)$ смешанного типа структур на многообразии с границей $[9$; c. 116].

Обозначим через $\mathbf{L}$. 1-связное накрытие спектра точки в алгебраической $L$ теории и через $\mathbf{L}\left(\pi_{1}(Y)\right)$ спектр многообразия $Y$ в алгебраической $L$-теории (см. [1; с. 544], [2] и [7; с. 28]). Мы строим отображение спектров

$$
\Omega^{q}\left(X_{+} \wedge \mathbf{L}_{\bullet}\right) \rightarrow \mathbf{L}\left(\pi_{1}(Y)\right),
$$

отождествляем гомотопическую группу $\pi_{n-q}(\mathbf{F})$ слоя $\mathbf{F}$ этого отображения со структурным множеством $\mathscr{N}_{n}(X, Y)$ и показываем, что имеет место изоморфизм

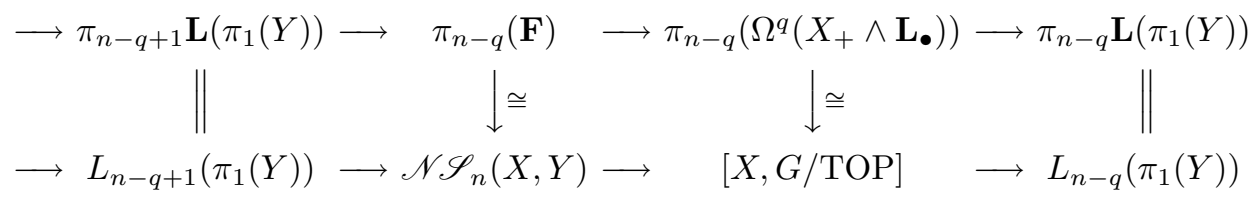

длинной точной последовательности гомотопических групп отображения

$$
\Omega^{q}\left(X_{+} \wedge \mathbf{L}_{\bullet}\right) \rightarrow \mathbf{L}\left(\pi_{1}(Y)\right),
$$

начинающегося с уровня $n-q$, с рассмотренной выше точной последовательностью (4) (теорема 2). Как тривиальное следствие теоремы получается, что $\mathscr{N} \mathscr{S}_{n}(X, Y)$ имеет групповую структуру, делающую (4) точной последовательностью групп. Доказаны пять других теорем, которые дают прямоугольные диаграммы и косы точных последовательностей гомотопических групп спектров, связывающие различными путями все группы, введенные выше.

Оставшаяся часть работы организована следующим образом. В $§ 2$ мы напоминаем общие конструкции и свойства спектров алгебраической $L$-теории, относящиеся к многообразию с подмногообразием. В $\S 3$ доказаны теоремы 1 и 2 , и в $\S 4$ получены диаграммы точных последовательностей, связывающие введенные выше группы. 


\section{§ 2. Спектры и $L$-группы}

Пусть $R$ - кольцо с инволюцией. В работе [1] (см. также [10] и [2]), используя квадратичные $n$-ады Пуанкаре над кольцом $R$, построен симплициальный $\Omega$ спектр $\mathbf{L}(R)=\left\{\mathbf{L}_{-k}(R) \mid k \in \mathbb{Z}\right\}$, для которого

$$
\mathbf{L}_{m+1}(R) \simeq \Omega \mathbf{L}_{m}(R), \quad \mathbf{L}_{m}(R) \simeq \mathbf{L}_{m+4}(R), \quad L_{n}(R)=\pi_{n}(\mathbf{L}(R))=\pi_{n}\left(\mathbf{L}_{0}(R)\right) .
$$

Если $\pi$ обозначает группу, снабженную гомоморфизмом $w: \pi \rightarrow\{ \pm 1\}$, обычно называемым гомоморфизмом ориентации, и если $\mathbb{Z} \pi$ обозначает целочисленное групповое кольцо, снабженное инволюцией, определяемой по правилу

$$
\sum a_{g} g \mapsto \sum a_{g} w(g) g^{-1}, \quad a_{g} \in \mathbb{Z}, \quad g \in \pi,
$$

то спектр $\mathbf{L}(\mathbb{Z} \pi)$ обозначается $\mathbf{L}(\pi)$. Если необходимо точно указать гомоморфизм ориентации $w$, мы будем писать $\mathbf{L}(\pi, w)$ вместо $\mathbf{L}(\pi)$.

В $[9 ; \S 17 \mathrm{~A}]$ приведена геометрическая конструкция спектра $\mathbf{L}(K) \simeq \mathbf{L}(\mathbb{Z} \pi)$ для топологического пространства $K$ с $\pi=\pi_{1}(K)$ и заданным гомоморфизмом $\pi_{1}(K) \rightarrow\{ \pm 1\}$. Нульмерными симплексами пространства $\mathbf{L}_{m}(K)$ являются объекты над $K$ размерности $m$ в смысле [9]. Напомним определение объекта размерности $m$ над пространством $K[9 ; \S 9]$. Пусть $(X, Y)$ - некоторая простая пара Пуанкаре с заданным расслоением $\nu$ над $X$ и $(M, \partial M)$ - компактное многообразие размерности $m$ с границей $\partial M$. Нормальное отображение степени 1

$$
f:(M, \partial M) \rightarrow(X, Y)
$$

для которого ограничение $\left.f\right|_{\partial M}: \partial M \rightarrow Y$ является простой гомотопической эквивалентностью, задает объект над $K$ размерности $m$, если задано стабильное оснащение расслоения $\tau_{M} \oplus f^{*} \nu$ (здесь $\tau_{M}$ - касательное расслоение к многообразию $M)$ и такое отображение $\omega: X \rightarrow K$, что гомоморфизм ориентации пространства $X$ разлагается в композицию

$$
\pi_{1}(X) \stackrel{\omega_{*}}{\longrightarrow} \pi_{1}(K) \rightarrow\{ \pm 1\} .
$$

Аналогично (см. $[9 ; \S 9]), \quad k$-симплексами комплекса $\mathbf{L}_{m}(K)$ являются объекты типа $k+1$ и размерности $m+k$ над $(k+1)$-адой $(K ; K, \ldots, K)$, для которой тотальное пространство и все подпространства совпадают с $K$ (см. [9; $§ 0]$. В высоких размерностях $(m \geqslant 6)$ гомотопический тип $\mathbf{L}_{m}(K)$ зависит только от $\pi_{1}(K)$.

Для любого морфизма $i: R \rightarrow T$ колец с инволюцией существует корасслоение спектров

$$
\cdots \longrightarrow \mathbf{L}(R) \longrightarrow \mathbf{L}(T) \longrightarrow \mathbf{L}(R \rightarrow T) \longrightarrow \cdots,
$$

гомотопическая длинная точная последовательность которого является относительной точной последовательностью $L$-групп отображения $i[10 ; \S 7]$ :

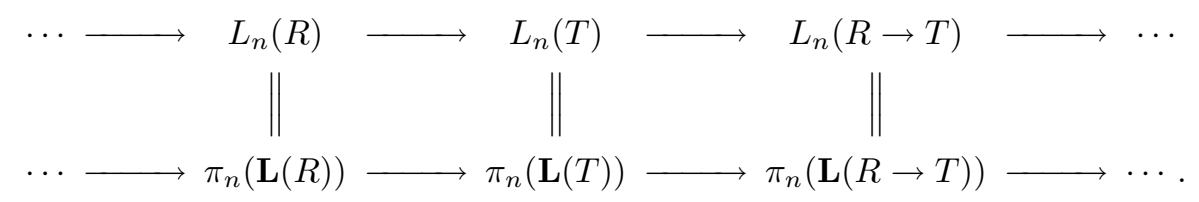


Пусть

$$
\left(D^{q}, S^{q-1}\right) \longrightarrow(U, \partial U) \stackrel{p}{\longrightarrow} Y
$$

обозначает дисковое расслоение над замкнутым многообразием $Y$. Поскольку отображение трансфера $L$-групп определено на уровне спектров (см. $[10 ; \S 7]$ и [9; с. 253]), имеет место коммутативная диаграмма

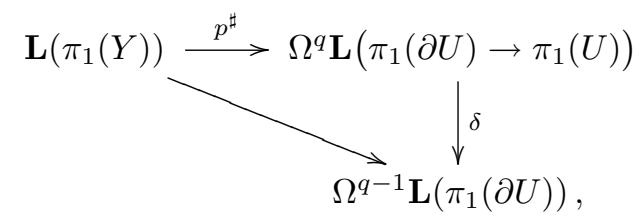

где $\delta$ - связывающее отображение в корасслоенной последовательности (5) спектров для естественного отображения фундаментальных групп $\pi_{1}(\partial U) \rightarrow$ $\pi_{1}(U)$.

Теперь пусть $Y \subset X$ является подмногообразием коразмерности $q$ связного замкнутого многообразия $X$. Трубчатая окрестность $U$ подмногообразия $Y$ в $X$ с границей $\partial U$ является дисковым расслоением над $Y$, и определен квадрат фундаментальных групп $F$ в (1). Имеется гомотопически коммутативная диаграмма спектров

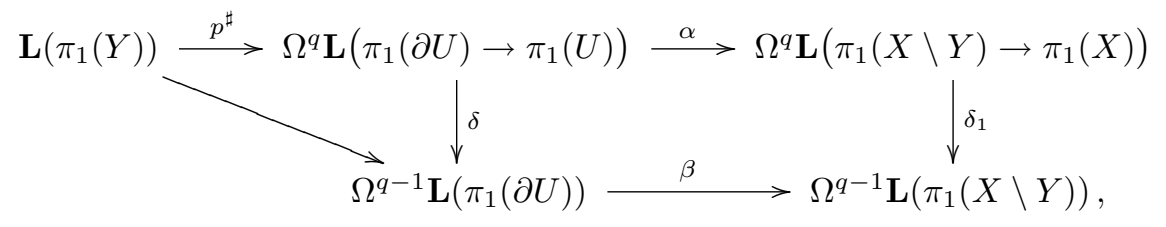

где левый треугольник тот же, что и в (6), а горизонтальные отображения правого квадрата индуцированы горизонтальными отображениями квадрата $F$.

Мы определяем спектр

$\mathbf{L S}(F)=$ homotopy cofiber $\left[\Omega\left(\alpha p^{\sharp}\right): \Omega \mathbf{L}\left(\pi_{1}(Y)\right) \rightarrow \Omega^{q+1} \mathbf{L}\left(\pi_{1}(X \backslash Y) \rightarrow \pi_{1}(X)\right)\right]$,

где $\alpha$ и $p^{\sharp}$ такие же, как в (7). Мы определяем спектр

$$
\mathbf{L P}(F)=\text { homotopy cofiber }\left[\Omega\left(\beta \delta p^{\sharp}\right): \Omega \mathbf{L}\left(\pi_{1}(Y)\right) \rightarrow \Omega^{q} \mathbf{L}\left(\pi_{1}(X \backslash Y)\right)\right],
$$

где $\beta, \delta$ и $p^{\sharp}$ такие же, как в (7) (см. [11]-[13] для специального случая подмногообразий).

Напомним, что в гомотопической категории спектров понятия универсальнопритягивающего и универсально-отталкивающего квадрата эквивалентны. Гомотопически коммутативный квадрат спектров является универсально-притягивающим тогда и только тогда, когда слои (а следовательно, и кослои) любых двух параллельных отображений естественно гомотопически эквивалентны. 
ПРЕДЛОЖЕНИЕ 1. Гомотопическая коммутативность (7) индуцирует c точностью до гомотопии такое отображение спектров $\mathbf{L S}(F) \rightarrow \mathbf{L P}(F)$, что диаграмма спектров

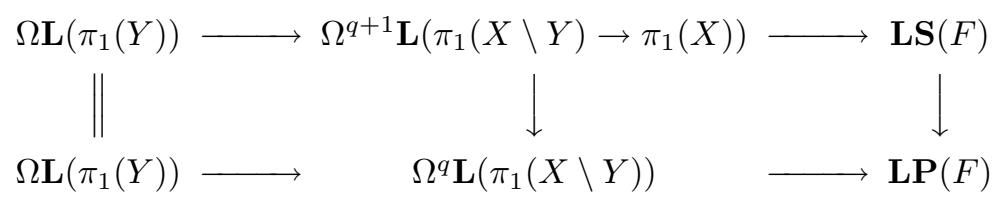

гомотопически коммутативна, горизонталъные строки являются корасслоениями и правый квадрат является универсально-притягивающим. Более того, если $L S_{n}(F)$ и $L P_{n}(F)$ обозначают соответственно группы препятствий $\kappa$ расщеплению и к перестройкам на $(X, Y)$, определенным в $[1 ; \S 7.2]$, то имеют место изоморфизмь

$$
\pi_{n}(\mathbf{L S}(F)) \cong L S_{n}(F), \quad \pi_{n}(\mathbf{L P}(F)) \cong L P_{n}(F)
$$

которые функториальны по F.

ДокАзАтЕЛЬство. Утверждения о диаграмме очевидны (см. [14]). Изоморфизмы для групп препятствий следуют из леммы о пяти гомоморфизмах и определения групп $L S_{*}$ и $L P_{*}$.

Замечание. Пусть $C=\pi_{1}(X \backslash Y)$ и $D=\pi_{1}(X)$. Тогда гомотопические длинные точные последовательности отображений в универсально-притягивающем квадрате предложения 1 составляются вместе, образуя косу точных последовательностей

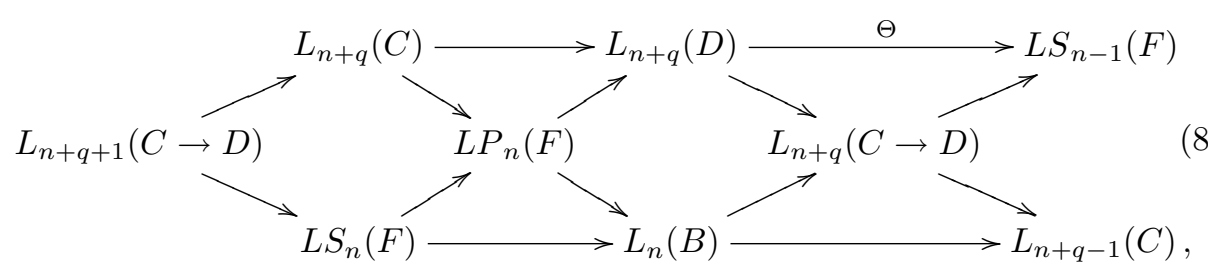

восходящую к Уоллу [9; с. 264].

\section{§ 3. Точная последовательность теории перестроек пары многообразий}

Для любого топологического пространства $X$ такого, что $\pi_{1}(X)=\pi$, существует алгебраическая точная последовательность теории перестроек (см. [1; с. 559$],[2 ;$ с. 278$]$ и $[9 ;$ с. 116])

$$
\cdots \longrightarrow L_{m+1}(\pi) \longrightarrow \mathbb{S}_{m+1}(X) \longrightarrow H_{m}\left(X ; \mathbf{L}_{\bullet}\right) \longrightarrow L_{m}(\pi) \longrightarrow \cdots
$$

Спектр $\mathbf{L}$ • является 1-связным накрытием $\Omega$-спектра кольца $\mathbf{L}(\mathbb{Z})$ и согласно [2; с. 291] (см. также [1; с. 545], [8; предложение 16.1] и [9; §17А]) $\mathbf{L}_{\bullet 0} \simeq G / \mathrm{TOP}$. Отображение

$$
\sigma_{*}: G / \mathrm{TOP} \rightarrow \mathbf{L}_{\bullet 0},
$$


задающее эту гомотопическую эквивалентность на симплициальном уровне, описано в [2; с. 291]. Это отображение сопоставляет сингулярному симплексу $s: \Delta \rightarrow G /$ ТОР квадратичную $n$-аду Пуанкаре над кольцом $\mathbb{Z}$ нормального отображения $n$-ад $(f, b): M \rightarrow \Delta$, которая классифицируется отображением $s$. По определению (9) является гомотопической длинной точной последовательностью корасслоения

$$
X_{+} \wedge \mathbf{L}_{\bullet} \rightarrow \mathbf{L}(\pi)
$$

спектров с гомотопическим слоем $\mathbf{S}(X)$, где

$$
\begin{aligned}
\mathbb{S}_{m+1}(X) & =\pi_{m}(\mathbf{S}(X)), \\
H_{m}\left(X ; \mathbf{L}_{\bullet}\right) & =\pi_{m}\left(X_{+} \wedge \mathbf{L}_{\bullet}\right), \\
L_{m}(\pi) & =\pi_{m}(\mathbf{L}(\pi))
\end{aligned}
$$

Если $X$ - замкнутое $n$-мерное топологическое многообразие, то точная последовательность (9) соответствует для $m \geqslant n$ точной последовательности теории перестроек, так как $H_{n}\left(X ; \mathbf{L}_{\bullet}\right)=[X, G / \mathrm{TOP}]$ и $\mathbb{S}_{n+1}(X)=\mathscr{S}_{n}^{s}(X)$. Точная последовательность теории перестроек продолжается влево (см. [2], [8; §18] и $[9 ; \S 10])$ следующим образом $(i \geqslant 1)$ :

$$
\begin{aligned}
\cdots \longrightarrow L_{n+i+1}\left(\pi_{1}\right) & \longrightarrow \mathscr{S}_{\partial}^{s}\left(X \times D^{i}\right) \\
& \longrightarrow\left[X \times D^{i}, \partial\left(X \times D^{i}\right) ; G / \mathrm{TOP}, *\right] \longrightarrow L_{n+i}\left(\pi_{1}\right) \longrightarrow \cdots,
\end{aligned}
$$

где $D^{i}-i$-мерный диск. Структурные множества $\mathscr{S}_{\partial}^{s}\left(X \times D^{i}\right)$ состоят из классов конкордантных относительно границы (см. [2; с. 559]) простых гомотопических эквивалентностей

$$
f:(M, \partial M) \rightarrow\left(X \times D^{i}, \partial\left(X \times D^{i}\right)\right),
$$

где $M$ - компактное многообразие и ограничение отображения $f$ на границу

$$
\left.f\right|_{\partial M}: \partial M \rightarrow \partial\left(X \times D^{i}\right)
$$

является гомеоморфизмом. Напомним, что конкордантность двух таких отображений

$$
f_{j}:\left(M_{j}, \partial M_{j}\right) \rightarrow\left(X \times D^{i}, \partial\left(X \times D^{i}\right)\right), \quad j=0,1,
$$

задается простой гомотопической эквивалентностью 4-ад

$$
\begin{aligned}
\left(h ; g, f_{0}, f_{1}\right):\left(W ; V, M_{0}, M_{1}\right) \rightarrow\left(\left(X \times D^{i}\right) \times I ; \partial\left(X \times D^{i}\right) \times I,\right. & \left.\left(X \times D^{i}\right) \times\{0\},\left(X \times D^{i}\right) \times\{1\}\right),
\end{aligned}
$$

где

$$
I=[0,1], \quad V=\partial M_{0} \times I, \quad \partial V=\partial M_{0} \cup \partial M_{1}
$$

и

$$
g=\partial f_{0} \times \operatorname{Id}: V \rightarrow \partial\left(X \times D^{i}\right) \times I
$$


Пусть $Y^{n-q} \subset X^{n}$ - подмногообразие коразмерности $q$ в $n$-мерном топологическом многообразии $X$. Тогда квадрат $F(1)$ фундаментальных групп определен. Из диаграммы (2) и алгебраической точной последовательности теории перестроек (9) (см. [9; с. 136]) мы получаем коммутативную диаграмму

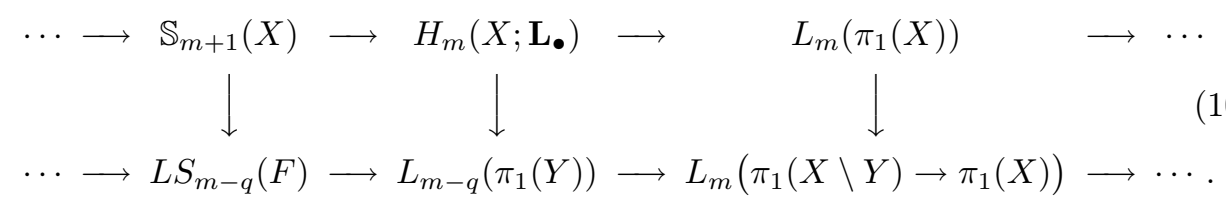

ПрЕДЛОЖЕНИЕ 2. Существует гомотопически коммутативный квадрат

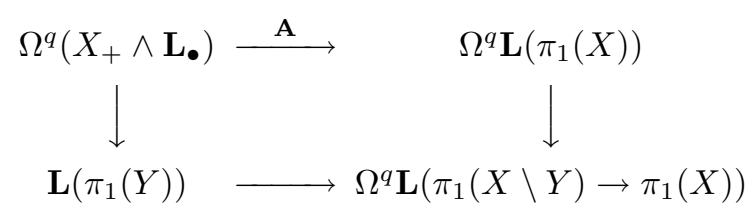

спектров такой, что коммутативная диаграмма (10) получается каноническим отображением гомотопической длинной точной последовательности верхнего отображения в (11) в такую же последовательность нижнего отображения в (11).

ДокАЗАТЕЛЬство. Пусть $U$ - трубчатая окрестность с границей $\partial U$ подмногообразия $Y$, и пусть $Z=X \backslash Y$. Определим коммутативную диаграмму

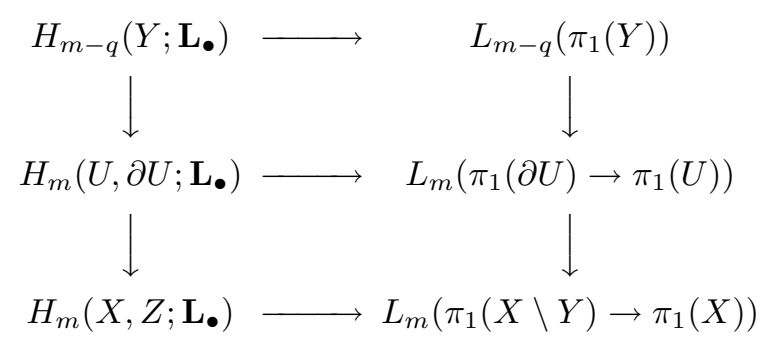

так, что горизонтальные отображения соответствуют сигнатурным отображениям, верхние вертикальные отображения являются отображениями трансфеpa (см. [1; с. 579]), а нижние вертикальные отображения являются каноническими. Квадрат $F$ индуцирует гомотопически коммутативную диаграмму спектров [14]

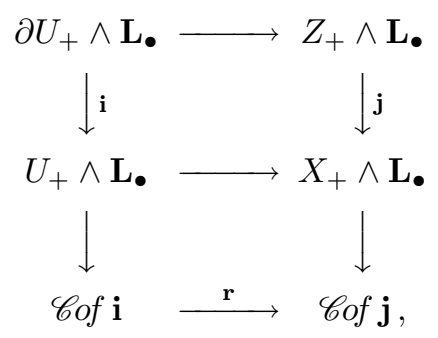


вертикальные столбцы которой являются корасслоениями. Для всех $m$ мы имеем коммутативную диаграмму

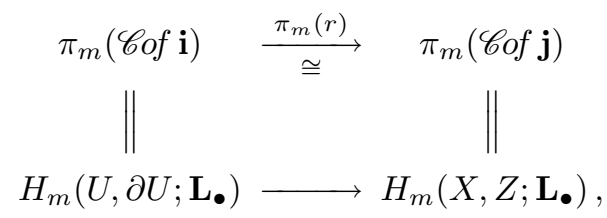

где равенства следуют по определению $H_{m}($; ), а нижнее горизонтальное отображение является изоморфизмом вырезания. Следовательно, отображение $\pi_{m}(r)$ является изоморфизмом и отображение $\mathbf{r}-$ слабая гомотопическая эквивалентность. Теперь из [1] следует, что $\mathbf{r}$ - гомотопическая эквивалентность. Согласно [1; с. 579] верхний квадрат диаграммы (12) реализуется на уровне спектров гомотопически коммутативной диаграммой спектров

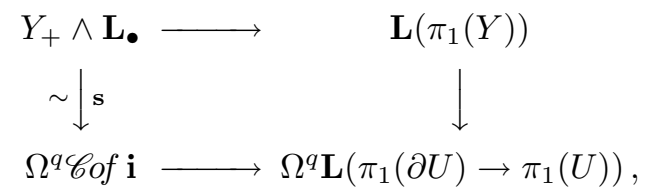

в которой левое вертикальное отображение является слабой гомотопической эквивалентностью [1; с. 579] и, следовательно, согласно [14] гомотопической эквивалентностью. В силу естественности сигнатурного отображения нижний квадрат диаграммы (12) реализуется на уровне спектров гомотопически коммутативной диаграммой спектров

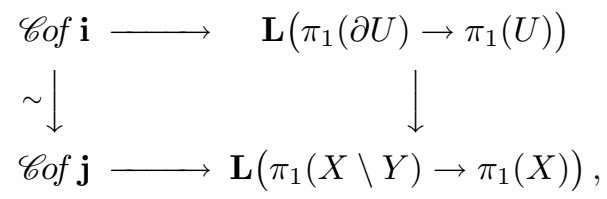

и согласно (14) левое вертикальное отображение является гомотопической эквивалентностью. Из диаграмм (15) и (16) мы получаем гомотопически коммутативную диаграмму

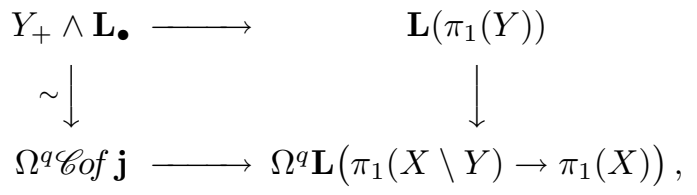

в которой левое вертикальное отображение является гомотопической эквивалентностью. В силу естественности сигнатурного отображения имеет место гомотопическая диаграмма спектров

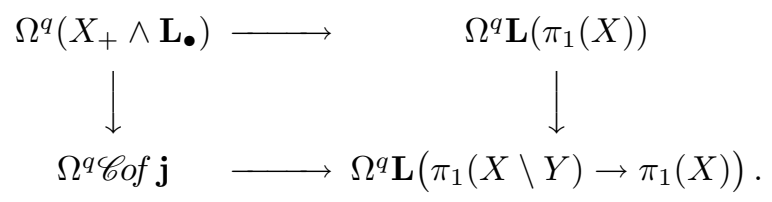

Согласно (17) нижнее горизонтальное отображение с точностью до гомотопии пропускается через $\mathbf{L}\left(\pi_{1}(Y)\right)$. Это доказывает предложение. 
Напомним определение нормального отображения в замкнутое топологическое многообразие $X[1$; с. 604]. Предположим, $X$ имеет размерность $n$. Тогда топологическое нормальное отображение $(f, b): M \rightarrow X$ состоит из следующего:

i) $n$-мерного многообразия $M$ с нормальным топологическим блочным расслоением

$$
\begin{gathered}
\nu_{M}=\nu_{M \subset S^{n+k}}: M \rightarrow \operatorname{BTOP}(k), \\
\rho_{M}: S^{n+k} \rightarrow S^{n+k} / \overline{S^{n+k}-E\left(\nu_{M}\right)}=T\left(\nu_{M}\right)
\end{gathered}
$$

ii) $n$-мерного многообразия $X$ с топологическим блочным расслоением

$$
\begin{aligned}
& \nu_{X}: X \rightarrow \operatorname{BTOP}(k), \\
& \rho_{X}: S^{n+k} \rightarrow T\left(\nu_{X}\right) ;
\end{aligned}
$$

iii) отображения степени один $f: M \rightarrow X$;

iv) отображения топологических блочных расслоений $b: \nu_{M} \rightarrow \nu_{X}$, накрывающего $f$, такого, что

$$
T(b)_{*}\left(\rho_{M}\right)=\rho_{X} \in \pi_{n+k}\left(T\left(\nu_{X}\right)\right) .
$$

Топологическое нормальное структурное множество многообразия $X$ является множеством классов конкордантности нормальных отображений $(f, b): M \rightarrow X$. Для $n \geqslant 5$ это множество совпадает с [X, G/TOP] (см. [1; с. 553-559]).

Пусть $f: M \rightarrow X-$ нормальное отображение, которое трансверсально подмногообразию $Y$. Пусть $N=f^{-1}(Y)$. Предположим, что ограничение $\left.f\right|_{N}$ : $N \rightarrow Y$ является простой гомотопической эквивалентностью. Два таких нормальных отображения $f_{i}: M_{i} \rightarrow X, i=1,2$, с $N_{i}=f_{i}^{-1}(Y)$ будут считаться эквивалентными, если существует нормальный кобордизм $F: W \rightarrow X$ со следующими свойствами:

i) $\partial W=M_{1} \cup M_{2}$ и $\left.F\right|_{M_{i}}=f_{i}, i=1,2$;

ii) $F$ трансверсально $Y$ с $F^{-1}(Y)=V$ и $\partial V=N_{1} \cup N_{2}$;

iii) ограничение $\left.F\right|_{V}$ является $s$-кобордизмом между $\left.F\right|_{N_{i}}=f_{i}, i=1,2$.

Обозначим классы эквивалентности таких отображений через $\mathscr{N}_{n}(X, Y)$, где $n=\operatorname{dim} X$.

Множество $\mathscr{N} \mathscr{S}_{n}(X, Y)$ имеет отмеченную точку, которая задается представителем $f:(M, N) \rightarrow(X, Y)$, где $\left.f\right|_{M}: M \rightarrow X-$ гомеоморфизм.

Существует естественное отображение

$$
\varphi: \mathscr{N}_{n}(X, Y) \rightarrow[X, G / \mathrm{TOP}]
$$

определяемое композицией забывающего отображения

$$
\mathscr{N} \mathscr{S}_{n}(X, Y) \rightarrow \mathscr{T}^{\mathrm{TOP}}(X)
$$

с отображением $\mathscr{T}^{\mathrm{TOP}} \rightarrow[X, G / \mathrm{TOP}]$, где $\mathscr{T}^{\mathrm{TOP}}(X)-$ множество классов конкордантности $t$-триангуляций $(f, b): M \rightarrow X$ (см. [1; с. 553-555]). 
Мы определим отображение (действие)

$$
\lambda: L_{n-q+1}\left(\pi_{1}(Y)\right) \rightarrow \mathscr{N} \mathscr{S}_{n}(X, Y)
$$

следующим образом. Пусть $f:(M, N) \rightarrow(X, Y)$ представляет некоторый класс $a \in \mathscr{N}_{n}(X, Y)$. Для любого элемента $x \in L_{n-q+1}\left(\pi_{1}(Y)\right)$ существует такое действие $x$ на ограничение $\left.f\right|_{N}: N \rightarrow Y$, что если $f_{1}=x\left(\left.f\right|_{N}\right): N_{1} \rightarrow Y$ является результатом этого действия, то $f_{1}$ - простая гомотопическая эквивалентность (см. [8; с. 196], [9; теоремы 5.6, 6.5 и § 10]).

Построение этого действия в [9] задает нормальное отображение многообразия с границей $G: V \rightarrow Y \times I$ с $\partial V=N \cup N_{1},\left.\quad G\right|_{N}=\left.f\right|_{N}: N \rightarrow Y \times\{0\}$ и $\left.G\right|_{N_{1}}=f_{1}: N_{1} \rightarrow Y \times\{1\}$. Препятствие к перестройке отображения $G$ относительно границы $\partial V$ равно $x \in L_{n-q+1}\left(\pi_{1}(Y)\right)$.

Пусть $E_{\xi}$ - трубчатая окрестность подмногообразия $Y$ в $X$ и $\partial E_{\xi}-$ граница этой окрестности. Отображение $f$ трансверсально подмногообразию $Y$ и $\left.F\right|_{N}: N \rightarrow Y$ - простая гомотопическая эквивалентность. Следовательно, $\left.f\right|_{N}$ индуцирует простую гомотопическую эквивалентность трубчатых окрестностей и их границ. Обозначим эту эквивалентность через

$$
g_{0}=\left.f\right|_{\left(E_{\nu}, \partial E_{\nu}\right)}:\left(E_{\nu}, \partial E_{\nu}\right) \rightarrow\left(E_{\xi}, \partial E_{\xi}\right)
$$

(см., например, [1; с. 579] и [9; с. 33]), где $E_{\nu}$ - трубчатая окрестность подмногообразия $N$ в $M$ и $\partial E_{\nu}$ - граница этой окрестности.

Отображение трансфера $p^{\sharp}$ спектров в диаграмме (6) индуцирует отображение трансфера $L$-групп

$$
p^{!}: L_{n-q+1}\left(\pi_{1}(Y)\right) \rightarrow L_{n+1}\left(\pi_{1}\left(\partial E_{\xi}\right) \rightarrow \pi_{1}\left(E_{\xi}\right)\right)
$$

и отображение трансфера

$$
p^{b}: \mathscr{S}_{n-q}(Y) \rightarrow \mathscr{S}_{n}\left(E_{\xi}, \partial E_{\xi}\right)
$$

структурных множеств.

Геометрическое определение отображения $p^{!}(x)$ (см. [1; с. 562-565] и [9; с. 133]) дает такое нормальное отображение многообразий с границами

$$
H: \Omega \rightarrow\left(E_{\xi}, \partial E_{\xi}\right) \times I,
$$

что $H$ трансверсально $Y \times I, \quad H^{-1}(Y \times I)=V$ и $\left.H\right|_{V}=G$. Ограничение отображения $H$ на нижнюю границу есть $g_{0}=\left.f\right|_{\left(E_{\nu}, \partial E_{\nu}\right)}$. Ограничение $H$ на верхнюю границу дает простую гомотопическую эквивалентность пар

$$
g_{1}:\left(E_{\eta}, \partial E_{\eta}\right) \rightarrow\left(E_{\xi}, \partial E_{\xi}\right),
$$

которая по определению является результатом действия $p^{!}(x)$ на простую гомотопическую эквивалентность $\left(\left.f\right|_{\left(E_{\nu}, \partial E_{\nu}\right)}\right)$. Ограничение $\left.g_{1}\right|_{N_{1}}$ есть $f_{1}$. В частности, элемент

$$
p^{b}\left(f_{1}: N_{1} \rightarrow Y\right) \in \mathscr{S}_{n}\left(E_{\xi}, \partial E_{\xi}\right)
$$

представляется простой гомотопической эквивалентностью пар

$$
g_{1}:\left(E_{\eta}, \partial E_{\eta}\right) \rightarrow\left(E_{\xi}, \partial E_{\xi}\right),
$$


которая трансверсальна подмногообразию $Y$ с $g_{1}^{-1}(Y)=N_{1}$. Кобордизм $\Omega$ и отображение $f: M \rightarrow X$ продолжаются до нормального кобордизма $F: W \rightarrow X$ с $\partial W=M \cup M_{1}$ [15; с. 45]. Ограничение $\left.F\right|_{M_{1}}$ определяет элемент $x(a) \in$ $\mathscr{N} \mathscr{S}_{n}(X, Y)$. Отметим, что для $x \neq 1$ отображение $F$ не определяет эквивалентности между $f=\left.F\right|_{M}$ и $\left.F\right|_{M_{1}}$ в вышеуказанном смысле, поскольку $V$ не является $s$-кобордизмом.

ЛЕмма 1. Определение отображения $\lambda$ корректно и является действием группы $L_{n-q+1}\left(\pi_{1}(Y)\right)$ на множестве $\mathscr{N} \mathscr{S}_{n}(X, Y)$.

ДокАзАтельство. Пусть $G^{\prime}: V^{\prime} \rightarrow Y$ обозначает такой другой нормальный кобордизм, построенный как и выше, что $\partial V^{\prime}=N \cup N_{1}^{\prime},\left.\quad G^{\prime}\right|_{N}=\left.f\right|_{N}$ и $\left.G^{\prime}\right|_{N_{1}^{\prime}}=f_{1}^{\prime}$. Пусть $F^{\prime}: W^{\prime} \rightarrow X$ обозначает такой нормальный кобордизм, продолжающий $G^{\prime}$ и $f: M \rightarrow X$, что $\partial W=M \cup M_{1}^{\prime}[15 ;$ с. 45]. Ограничение $\left.F\right|_{M_{1}^{\prime}}$ является другим представителем элемента $x(a) \in \mathscr{N} \mathscr{S}_{n}(X, Y)$. Мы докажем, что представители $\left.F\right|_{M_{1}}$ и $\left.F^{\prime}\right|_{M_{1}^{\prime}}$ эквивалентны в $\mathscr{N}_{n}(X, Y)$. Из [9; $\S 10.4]$ следует, что существует отображение нормального кобордизма $\Psi: \Lambda \rightarrow Y$ с нижний краем $\left.f\right|_{N} \times I: N \times I \rightarrow Y$, с левой стороной $G: V \rightarrow Y$, с правой стороной $G^{\prime}: V^{\prime} \rightarrow Y$ и с верхним краем, являющимся нормальным $s$-кобордизмом между $\left.G\right|_{N_{1}}: N_{1} \rightarrow Y$ и $\left.G^{\prime}\right|_{N_{1}^{\prime}}: N_{1}^{\prime} \rightarrow Y$. Пусть $W \cup_{\tau} M \times I \cup_{\tau^{\prime}} W^{\prime}$ обозначает пространство, полученное посредством очевидных приклеивающих отображений $\tau: M \times\{0\} \rightarrow W$ и $\tau^{\prime}: M \times\{1\} \rightarrow W^{\prime}$. Пусть

$$
H=F \cup(f \times I) \cup F^{\prime}: W \cup_{\tau} M \times I \cup_{\tau^{\prime}} W^{\prime} \rightarrow X .
$$

Пусть

$$
\rho: V \cup_{\left.\tau\right|_{N \times\{0\}}} N \times I \cup_{\left.\tau^{\prime}\right|_{N \times\{1\}}} W^{\prime} \rightarrow W \cup_{\tau} M \times I \cup_{\tau^{\prime}} W^{\prime}
$$

обозначает каноническое вложение, и используем $\rho$ для приклеивания $\Lambda$ к $W \cup_{\tau}$ $M \times I \cup_{\tau^{\prime}} W^{\prime}$ для получения пространства

$$
\widetilde{\Lambda}:=\Lambda \cup_{\rho}\left(W \cup_{\tau} M \times I \cup_{\tau^{\prime}} W^{\prime}\right)
$$

и отображения

$$
\Psi \cup_{\rho}(H): \widetilde{\Lambda} \rightarrow X
$$

Согласно [15; с. 45] мы можем продолжить $\Psi \cup_{\rho}(H)$ до нормального кобордизма $\Theta: \Omega \rightarrow X$, чье основание есть $f \times I: M \times I \rightarrow X$ и чей верх есть эквивалентность в определенном выше смысле между $f_{1}: M_{1} \rightarrow X$ и $f_{1}^{\prime}: M_{1}^{\prime} \rightarrow X$. Используя те же аргументы, как и в определении отображения $\lambda$, мы можем добиться, чтобы ограничение отображения $\Theta$ на верхнюю границу было трансверсально подмногообразию $Y$. Доказательство того, что мы имеем действие группы, вытекает стандартно из того факта, что действие $L_{n-q+1}\left(\pi_{1}(Y)\right)$ на $\mathscr{S}_{n-q}(Y)$ является действием группы $[9 ; \S 10]$.

Существует естественное геометрическое отображение

$$
\alpha_{1}:\left[X \times D^{1}, X \times S^{0} ; G / \mathrm{TOP}, *\right] \rightarrow L_{n-q+1}\left(\pi_{1}(Y)\right),
$$

получаемое композицией отображения ограничения

$$
\left[X \times D^{1}, X \times S^{0} ; G / \mathrm{TOP}, *\right] \rightarrow\left[Y \times D^{1}, Y \times S^{0} ; G / \mathrm{TOP}, *\right]
$$


с отображением

$$
\left[Y \times D^{1}, Y \times S^{0} ; G / \mathrm{TOP}, *\right] \rightarrow L_{n-q+1}\left(\pi_{1}(Y)\right) .
$$

Аналогично, имеет место естественное геометрическое отображение

$$
\alpha:[X ; G / \mathrm{TOP}] \rightarrow L_{n-q+1}\left(\pi_{1}(Y)\right) .
$$

Теорема 1. Последователъность

$$
\begin{gathered}
\cdots \longrightarrow\left[X \times D^{1}, X \times S^{0} ; G / \mathrm{TOP}, *\right] \stackrel{\alpha_{1}}{\longrightarrow} L_{n-q+1}\left(\pi_{1}(Y)\right) \\
\stackrel{\lambda}{\longrightarrow} \mathcal{N}_{n}(X, Y) \stackrel{\varphi}{\longrightarrow}[X, G / \mathrm{TOP}] \stackrel{\alpha}{\longrightarrow} L_{n-q}\left(\pi_{1}(Y)\right)
\end{gathered}
$$

точна.

ДоказАТЕЛЬство. Точность в $[X, G / \mathrm{TOP}]$. Существует естественное отображение $\mathscr{N}_{n}(X, Y) \rightarrow \mathscr{S}_{n-q}(Y)$, получаемое ограничением на $Y$. Диаграмма

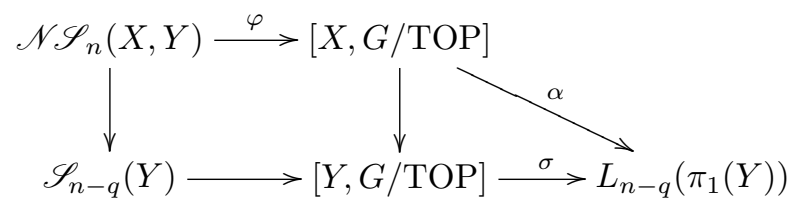

коммутативна, и нижняя строка точна согласно [1; предложение 7.1.4]. Таким образом, $\alpha \varphi=0$. Пусть $f: M \rightarrow X$ обозначает нормальное отображение, определяющее такой класс в $[X, G / \mathrm{TOP}]$, что $\alpha(f)=0$. Тогда $\sigma\left(\left.f\right|_{N}\right)=0$ и $\left.f\right|_{N}$ нормально кобордантно, скажем посредством $G: V \rightarrow Y$, нормальному отображению $g: N_{1} \rightarrow Y$, которое является простой гомотопической эквивалентностью. Согласно [15; с. 45] мы можем продолжить $f \cup G: M \cup V \rightarrow X$ таким нормальным кобордизмом $F: W \rightarrow X$, что $\left.F\right|_{N_{1}}=g$ является простой гомотопической эквивалентностью. Используя ту же последовательность рассуждений, как и в определении отображения $\lambda$, мы можем добиться, чтобы ограничение отображения $\left.F\right|_{M_{1}}$ на верхнюю границу стало трансверсально к подмногообразию $Y$. По определению класс $\left.F\right|_{M_{1}}$ лежит в образе $\varphi$.

Точность в $\mathscr{N}_{n}(X, Y)$. Если $x \in L_{n-q+1}\left(\pi_{1}(Y)\right)$ и $f:(M, N) \rightarrow(X, Y)$ представляет элемент из $\mathscr{N} \mathscr{S}_{n}(X, Y)$, то нормальное отображение $x(f)$ является по определению нормально кобордантным $f$ и тогда $f$ и $x(f)$ имеют один и тот же образ в $[X, G / \mathrm{TOP}]$. Это показывает по определению, что $\varphi \lambda=0$. Пусть

$$
f_{i}:\left(M_{i}, N_{i}\right) \rightarrow(X, Y), \quad i=1,2,
$$

- нормальные отображения, представляющие элементы из $\mathscr{N}_{n}(X, Y)$. Если $f_{i}$ представляют один и тот же элемент из $[X, G / \mathrm{TOP}]$, то существует по определению нормальный кобордизм $F^{\prime}: W^{\prime} \rightarrow X$ от $f_{1}$ к $f_{2}$. Поскольку $\left.f_{1}\right|_{N_{1}}$ и $\left.f_{2}\right|_{N_{2}}$ очевидно совпадают в $[Y, G / \mathrm{TOP}]$, то согласно $[9 ; \S 10]$ имеется такой элемент $x \in L_{n-q+1}\left(\pi_{1}(Y)\right)$, что $x\left(\left.f_{1}\right|_{N_{1}}\right)$ является нормально $s$-кобордантным $\left.f_{2}\right|_{N_{2}}$. Пусть $F: W \rightarrow X-$ нормальный кобордизм, построенный в определении $x\left(f_{1}\right)$. Мы хотим показать, что $x\left(f_{1}\right)$ эквивалентен $f_{2}$ в смысле, определенном 
перед леммой 1. Пусть $\Psi: \Lambda \rightarrow Y-$ нормальный кобордизм, соответствующий рисунку, данному в $[9 ; \S 10]$. Это соответствует нормальному кобордизму $\Psi: \Lambda \rightarrow Y$ в доказательстве леммы 1 . Теперь, как и в доказательстве этой леммы, существует нормальный кобордизм $\Theta: \Omega \rightarrow X$, продолжающий $\Psi$. Верх этого кобордизма является таким нормальным кобордизмом $\mathscr{J}$ от $x\left(f_{1}\right)$ к $f_{2}$, что $\left.\mathscr{J}\right|_{\mathscr{J}^{-1}(Y)}$ является нормальным $s$-кобордизмом от $x\left(\left.f_{1}\right|_{N_{1}}\right)$ к $\left.f_{2}\right|_{N_{2}}$. Как и в определении отображения $\lambda$, мы можем добиться того, чтобы отображение $\mathscr{J}$ стало трансверсально к подмногообразию $Y$.

Точность в $L_{n-q+1}\left(\pi_{1}(Y)\right)$. Пусть $x \in$ image $\left(\alpha_{1}\right)$, и пусть $f:(M, N) \rightarrow(X, Y)$ представляет элемент из $\mathscr{N} \mathscr{S}_{n}(X, Y)$. Пусть $G$ обозначает нормальный кобордизм в определении $x(f)$. По определению нижним отображением в $G$ является $\left.f\right|_{N}$, а верхним $-\left.x(f)\right|_{N_{1}}$. Поскольку диаграмма

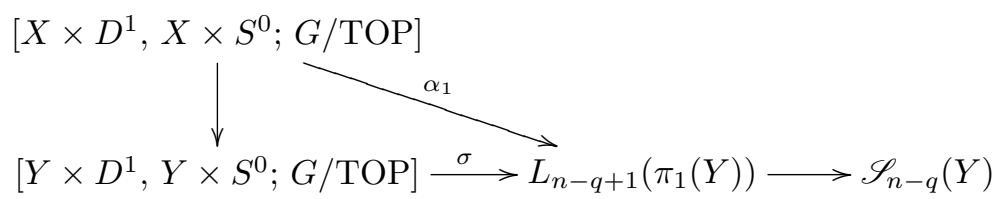

коммутативна и нижняя строка точна согласно [1; предложение 7.1.4] и [9; предложение 10.8], получаем, что $G$ является $s$-кобордизмом согласно [9; предложение 10.4]. Таким образом, кобордизм $F$ в определении $x(f)$ является эквивалентностью между $f$ и $x(f)$ в смысле, определенном перед леммой 1 . Пусть $x \in L_{n-q+1}\left(\pi_{1}(Y)\right)$ и $f \in \mathscr{N}_{n}(X, Y)$ таковы, что $f$ эквивалентно $x(f)$. Пусть $F:(W, V) \rightarrow(X, Y) \times I$ обозначает нормальное отображение, построенное в определении действия $x$ на $f:(M, N) \rightarrow(X, Y)$, и пусть $f_{1}:\left(M_{1}, N_{1}\right) \rightarrow$ $(X, Y)$ обозначает верхнее отображение в $F$. Пусть $G: V \rightarrow Y \times I$ обозначает ограничение $F$ на $F^{-1}(Y)$. Пусть $F^{\prime}:\left(W^{\prime}, V^{\prime}\right) \rightarrow(X, Y) \times I$ обозначает нормальное отображение, определяющее эквивалентность от $x(f)$ к $f$. Здесь нижним отображением будет $f_{1}:\left(M_{1}, N_{1}\right) \rightarrow(X, Y)$, а верхним отображением $-f:(M, N) \rightarrow(X, Y)$. Пусть $G^{\prime}: V^{\prime} \rightarrow Y \times I$ обозначает ограничение $F^{\prime}$ на $\left(F^{\prime}\right)^{-1}(Y)$. По определению $\left(F^{\prime}\right)^{-1}(Y)=V^{\prime}$ является $s$-кобордизмом. Мы отождествим верхнюю границу $W$ с нижней границей $W^{\prime}$ и получим новое нормальное отображение $H:(\Omega, \Lambda) \rightarrow(X, Y) \times I$, чьи нижнее и верхнее отображения совпадают с $f:(M, N) \rightarrow(X, Y)$. Следовательно, нормальное отображение $H$ лежит в $\left[X \times D^{1}, Y \times S^{0} ; G / \mathrm{TOP}, *\right]$. Нормальное отображение $\left.H\right|_{\Lambda}: \Lambda \rightarrow Y \times I$ очевидно является объединением нормальных отображений, заданных $G$ и $G^{\prime}$, и имеет элемент $x \in L_{n-q+1}\left(\pi_{1}(Y)\right)$ в качестве препятствия к перестройке относительно границы в силу аддитивности препятствий к перестройкам. Таким образом, $\alpha_{1}(H)=x$.

Пусть $\mathbf{F}$ обозначает гомотопический слой отображения

$$
\Omega^{q}\left(X_{+} \wedge \mathbf{L}_{\bullet}\right) \rightarrow \mathbf{L}\left(\pi_{1}(Y)\right)
$$

в квадрате (11). Пусть $\mathbf{N S}(X, Y)$ обозначает $q$-е распетливание $\mathbf{F}$. Отождествим $\mathbf{F}=\Omega^{q} \mathbf{N S}(X, Y)$. Пусть

$$
\mathbb{N S}_{m}(X, Y)=\pi_{m}(\mathbf{N S}(X, Y)),
$$


и пусть

$$
\cdots \longrightarrow \mathbb{N S}_{m}(X, Y) \longrightarrow H_{m}\left(X ; \mathbf{L}_{\bullet}\right) \longrightarrow L_{m-q}\left(\pi_{1}(Y)\right) \longrightarrow \cdots
$$

обозначает гомотопическую длинную точную последовательность отображения

$$
\Omega^{q}\left(X_{+} \wedge \mathbf{L}_{\bullet}\right) \rightarrow \mathbf{L}\left(\pi_{1}(Y)\right)
$$

ТЕОРема 2. Используем гомоморфизм групп

$$
L_{n-q+1}\left(\pi_{1}(Y)\right) \rightarrow \mathbb{N S}_{n}(X, Y)
$$

в (20), чтобъ снабдить $\mathbb{N S}_{n}(X, Y)$ левым действием посредством умножения в группе $L_{n-q+1}\left(\pi_{1}(Y)\right)$. Тогда имеет место изоморфизм

$$
\mathbb{N S}_{n}(X, Y) \rightarrow \mathscr{N} \mathscr{S}_{n}(X, Y)
$$

$L_{n-q+1}\left(\pi_{1}(Y)\right)$-действий, делающий диаграмму

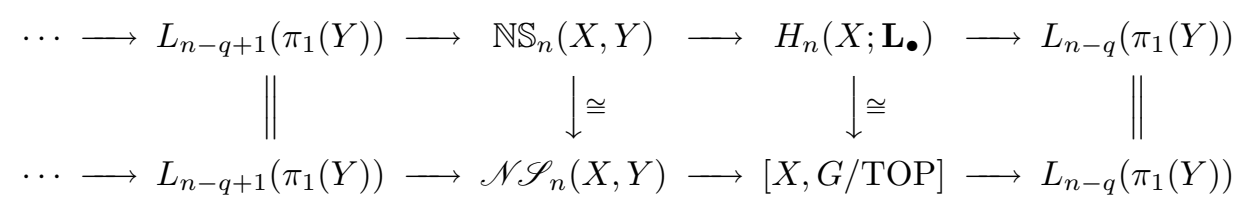

коммутативной. В частности, имеется групповая структура на $\mathscr{N}_{n}(X, Y)$, делающая (19) точной последовательностью групп.

ДокАЗАТЕЛЬСтво. Согласно [9; с. 116, 117 и 136] и [1; § 7.2] имеют место коммутативные квадраты

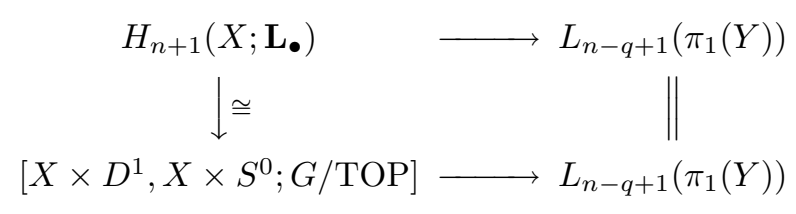

И

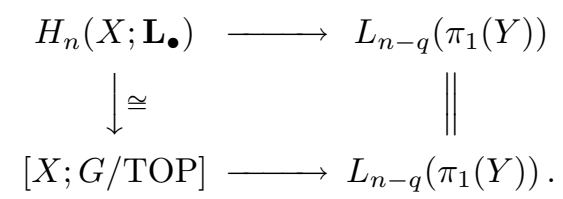

Элементарные выкладки, использующие эти квадраты и точные последовательности в $(20)$ и (19), дают требуемый изоморфизм $L_{n-q+1}\left(\pi_{1}(Y)\right)$-действий. Заключительное утверждение теоремы тривиально. 


\section{§ 4. Алгебраические свойства групп $\mathscr{N S}_{n}(X, Y)$}

Определим спектр $\Omega^{q} \mathbf{S}(X, Y, \xi)$ как гомотопический слой естественного отображения

$$
\Omega^{q} \mathbf{S}(X) \rightarrow \mathbf{L S}(F)
$$

Поскольку $\mathbf{S}(X)$ определяется как гомотопический слой $X_{+} \wedge \mathbf{L}_{\bullet} \rightarrow \mathbf{L}\left(\pi_{1}(X)\right)$, получаем, что $\Omega^{q} \mathbf{S}(X)$ является слоем верхнего отображения в (11). Спектр $\mathbf{L S}(F)$ является гомотопическим слоем нижнего отображения в квадрате (11) согласно [14; §8.32]. Мы получаем корасслоенную последовательность

$$
\Omega^{q} \mathbf{S}(X, Y, \xi) \longrightarrow \Omega^{q} \mathbf{S}(X) \longrightarrow \mathbf{L S}(F)
$$

Согласно $[1 ; \S 7]$ имеет место изоморфизм

$$
\pi_{m}(\mathbf{S}(X, Y, \xi))=\mathbb{S}_{m+1}(X, Y, \xi)
$$

и группы $\mathbb{S}_{m}(X, Y, \xi)$ входят в точную последовательность [1; предложение 7.2.6]

$$
\cdots \longrightarrow \mathbb{S}_{m+q+1}(X, Y, \xi) \longrightarrow \mathbb{S}_{m+q+1}(X) \longrightarrow L S_{m}(F) \longrightarrow \cdots
$$

Согласно [14; $\S 8.31]$ и [12; лемма 1] гомотопически коммутативный квадрат в (11) можно продолжить до бесконечной гомотопически коммутативной диаграммы спектров

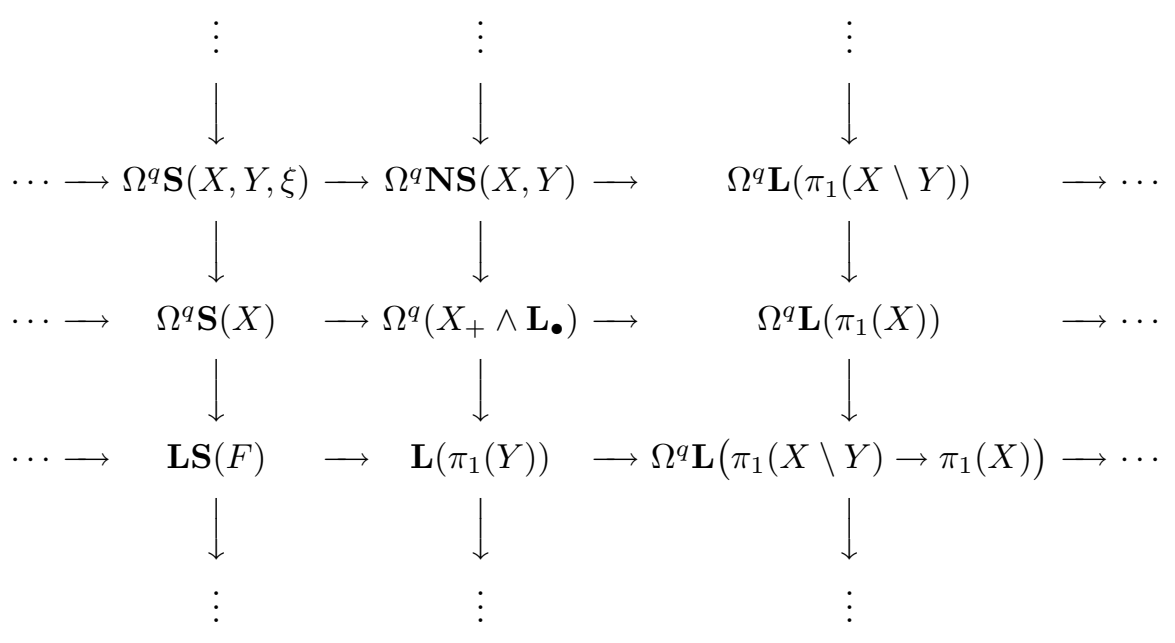

в которой каждые строка и столбец являются корасслоенными последовательностями. 
Теорема 3. Применяя $\pi_{0} \kappa(22)$, мы получаем бесконечный коммутативный комплекс групп

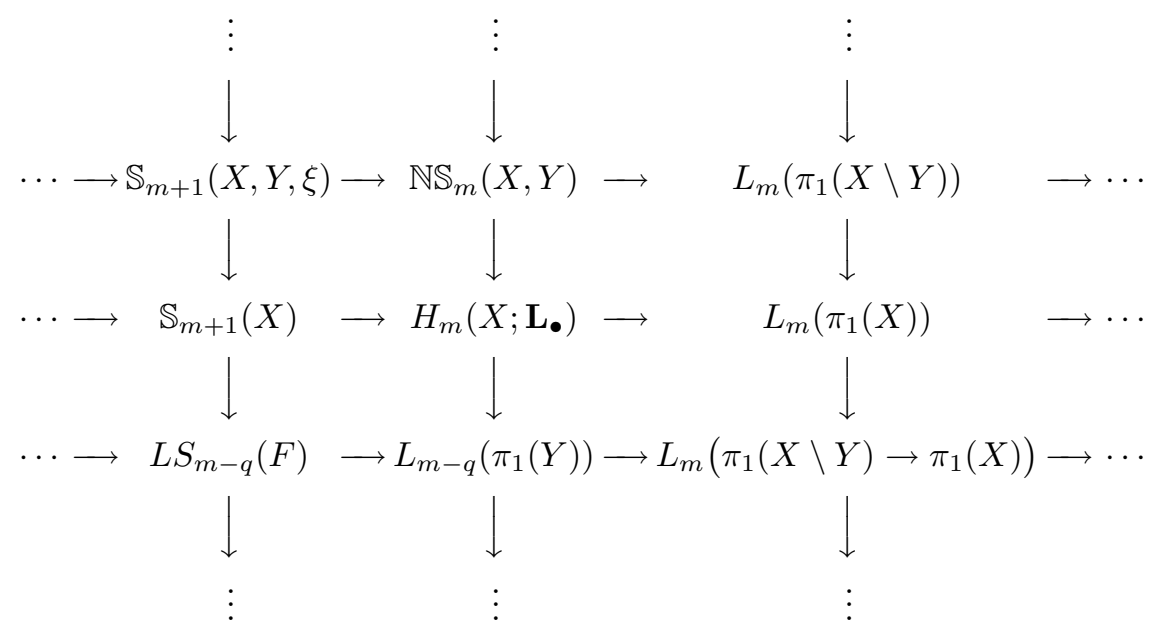

в котором все строки и столбцы точны.

ДокАЗАТЕльство. Поскольку комплекс в (22) является гомотопически коммутативным, получаем, что комплекс в теореме является коммутативным. Поскольку столбцы и строки в (22) являются корасслоенными последовательностями, получаем, что все строки и столбцы в (23) точны.

ЛЕмма 2. Пусть

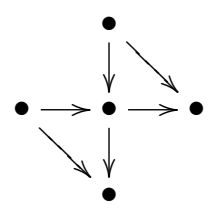

обозначает гомотопически коммутативную диаграмму, в которой строка и столбеи, являются корасслоениями. Тогда кослои наклонных отображений естественно гомотопически эквивалентны.

ДокАЗАТЕЛЬСТво см. в [12].

Бесконечная гомотопически коммутативная диаграмма (22) содержит гомотопически коммутативную диаграмму спектров

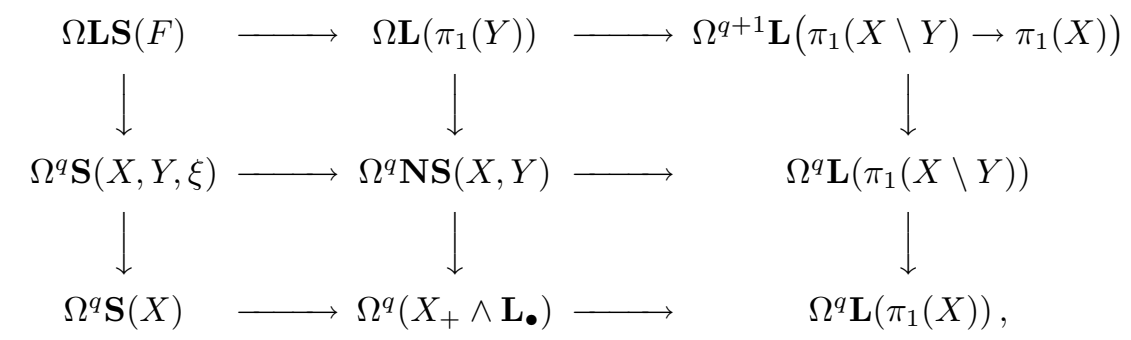


в которой все строки и столбцы являются корасслоениями. По лемме 2 кослой отображения

$$
\Omega^{q} \mathbf{S}(X, Y, \xi) \rightarrow \Omega^{q}\left(X_{+} \wedge \mathbf{L}_{\bullet}\right),
$$

полученный из диаграммы (24), естественно гомотопически эквивалентен кослою отображения

$$
\Omega \mathbf{L}\left(\pi_{1}(Y)\right) \rightarrow \Omega^{q} \mathbf{L}\left(\pi_{1}(X \backslash Y)\right),
$$

полученного из диаграммы (24). Но последний кослой является спектром $\mathbf{L P}(F)$ по определению. Таким образом, мы получаем гомотопически коммутативную диаграмму спектров

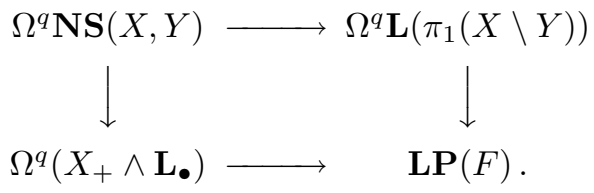

Поскольку гомотопические слои верхнего и нижнего отображений в (25) одинаковы, а именно равны $\Omega^{q} \mathbf{S}(X, Y, \xi)$, получаем, что (25) является универсальнопритягивающим и, следовательно, универсально-отталкивающим гомотопически коммутативным квадратом спектров.

TEOPEMA 4. Пусть

$$
\mathbb{N S}_{m+q}=\mathbb{N S}_{m+q}(X, Y), \quad \mathbb{S}_{m+q}(\xi)=\mathbb{S}_{m+q}(X, Y, \xi) .
$$

Тогда коса точных последовательностей, задаваемая (25), есть

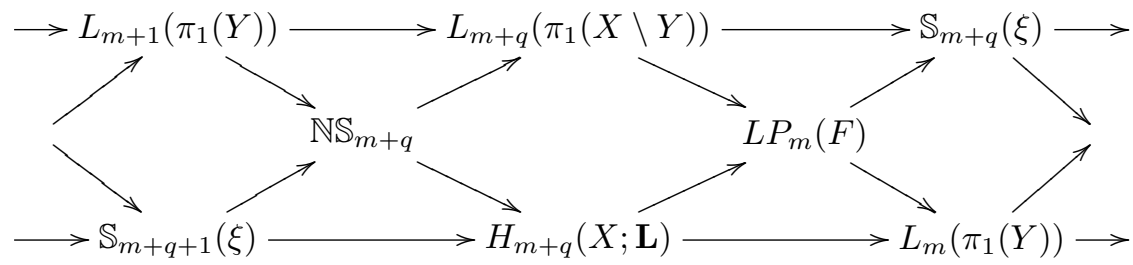

Бесконечная в двух направлениях диаграмма (22) содержит гомотопически коммутативную поддиаграмму спектров

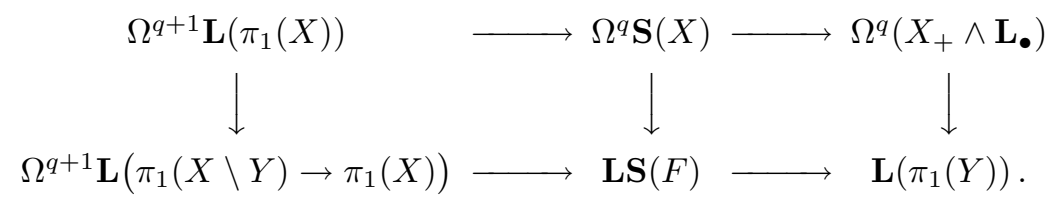

Поскольку каждая строка в этой диаграмме является корасслоенной последовательностью, получаем, что правое вертикальное отображение $\Omega^{q}\left(X_{+} \wedge \mathbf{L}_{\bullet}\right) \rightarrow$ $\mathbf{L}\left(\pi_{1}(Y)\right)$ в этой диаграмме пропускается с точностью до гомотопии через гомотопический кослой отображения $\Omega^{q+1} \mathbf{L}\left(\pi_{1}(X)\right) \rightarrow \mathbf{L} \mathbf{S}(F)$. Но по лемме 2 и из бесконечной в двух направлениях диаграммы (22) имеем

homotopy cofiber $\left[\Omega^{q+1} \mathbf{L}\left(\pi_{1}(X)\right) \rightarrow \mathbf{L S}(F)\right]$

$\sim$ homotopy cofiber $\left[\Omega^{q} \mathbf{S}(X, Y, \xi) \rightarrow \Omega^{q}\left(X_{+} \wedge \mathbf{L}_{\bullet}\right)\right]$

$\sim$ homotopy cofiber $\left[\Omega \mathbf{L}\left(\pi_{1}(Y)\right) \rightarrow \Omega^{q} \mathbf{L}\left(\pi_{1}(X \backslash Y)\right)\right] \stackrel{\text { def }}{=} \mathbf{L P}(F)$. 
Таким образом, мы получаем гомотопически коммутативную диаграмму спектров

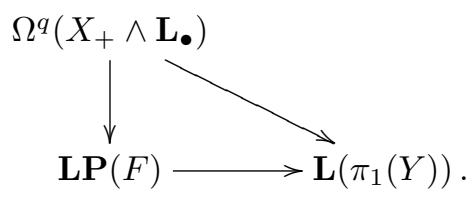

Определение отображения $\Omega^{q}\left(X_{+} \wedge \mathbf{L}_{\bullet}\right) \rightarrow \mathbf{L}\left(\pi_{1}(Y)\right)$ дано после (18) в доказательстве предложения 2. Оно является композицией четырех отображений

$$
\Omega^{q}\left(X_{+} \wedge \mathbf{L}_{\bullet}\right) \longrightarrow \Omega^{q} \mathscr{C o f} \mathbf{j} \stackrel{\sim}{\longrightarrow} Y_{+} \wedge \mathbf{L}_{\bullet} \longrightarrow \mathbf{L}\left(\pi_{1}(Y)\right)
$$

Так мы можем продолжить диаграмму, полученную выше, до гомотопически коммутативной диаграммы спектров

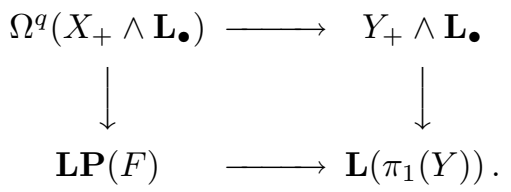

Как и в доказательстве теоремы 3, мы можем сопоставить (27) бесконечную в двух направлениях гомотопически коммутативную диаграмму и применить $\pi_{0}$ к этой диаграмме, чтобы получить бесконечную в двух направлениях коммутативную диаграмму групп

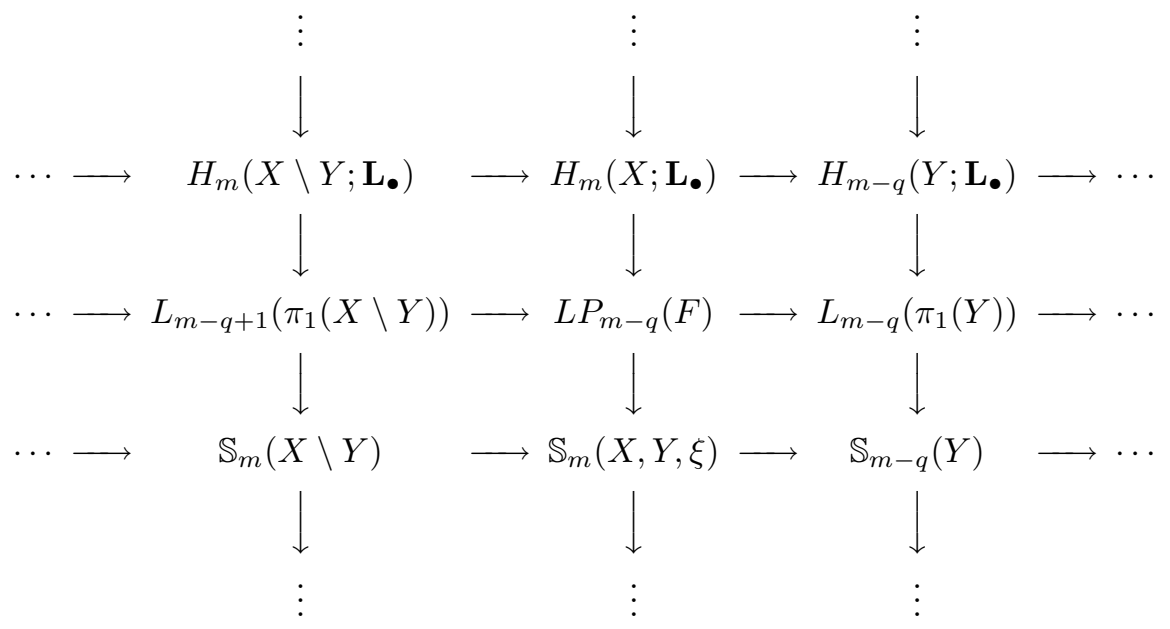

строки и столбцы которой точны. Это диаграмма из [1; предложение 7.2.6], и гомотопически коммутативный квадрат (27) служит для реализации бесконечной в двух направлениях коммутативной диаграммы (28) на уровне спектров.

Теорема 5. Как и в доказательстве теоремь 4, мы можем сопоставить гомотопически коммутативному квадрату (27) бесконечную в двух направлениях гомотопически коммутативную диаграмму, строки и столбцы которой 
являются корасслоенными последовательностями, и получить из диаграммы гомотопический универсально-отталкивающий квадрат, который в данной ситуации есть

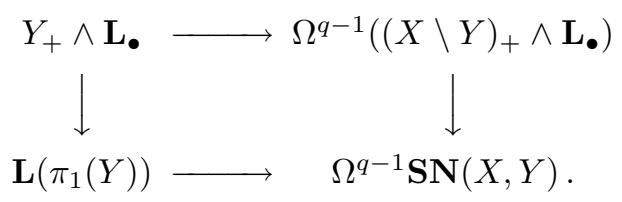

Коса точных последовательностей, определяемая (29), есть

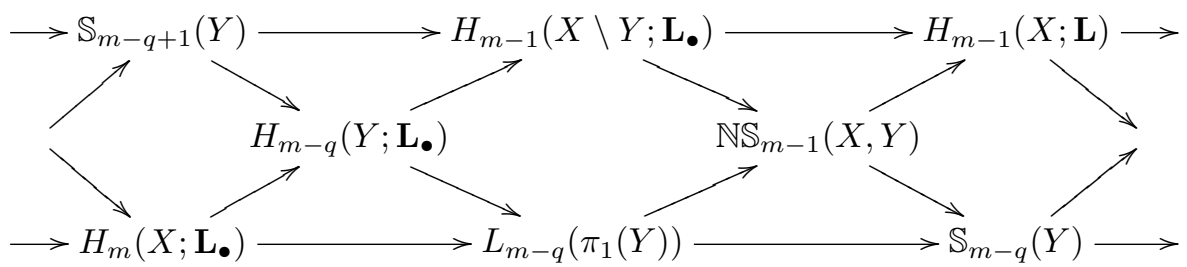

ТЕОРема 6. Как и в доказательстве теоремы 4, мы можем сопоставить гомотопически коммутативному квадрату (27) бесконечную в двух направлениях гомотопически коммутативную диаграмму, строки и столбцы которой являются корасслоенными последовательностями, и получить из диаграммы гомотопический универсально-отталкивающий квадрат, который в данной ситуации есть

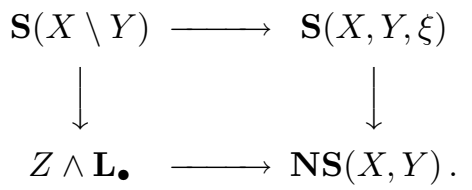

Коса точных последовательностей, определяемая (30), есть

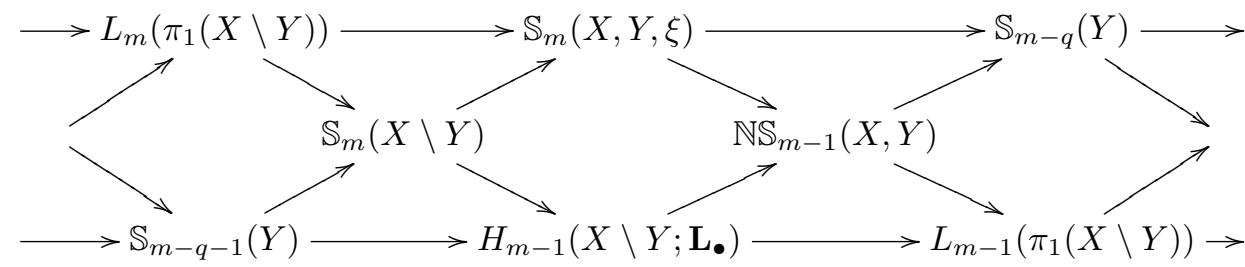

Пусть $Y \subset X \subset W$ - тройка замкнутых топологических многообразий такая, что $n$ - размерность $X, q$ - коразмерность $Y$ в $X$ и $q^{\prime}-$ коразмерность $X$ в $W$. Мы будем предполагать, что каждое подмногообразие является локально плоским в объемлющем многообразии и удовлетворяет условиям, сформулированным в [8; с. 570] для пары многообразий. В этом случае мы можем описать соотношения между группами $\mathbb{N S}_{*}(W, Y), \mathbb{N S}_{*}(X, Y)$ и множествами нормальных инвариантов многообразий $X, W$ и $W \backslash X$. 
ТеОрема 7. Существует коса точных последовательностей

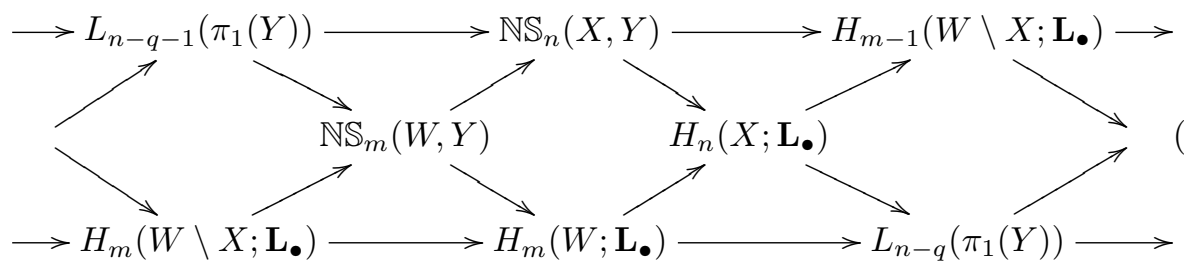

где $m=n+q^{\prime}-$ размерность многообразия $W$.

ДокАЗАТЕЛЬСтво. Рассмотрим гомотопически коммутативный квадрат спектров

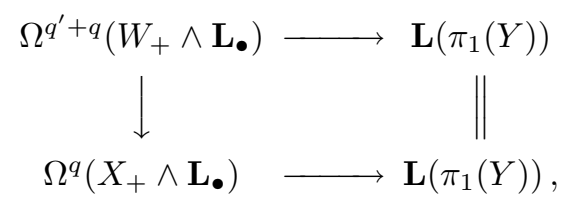

где левое вертикальное отображение является реализацией на уровне спектров композиции отображения

$$
H_{m}\left(W ; \mathbf{L}_{\bullet}\right) \longrightarrow H_{m}\left(W, W \backslash X ; \mathbf{L}_{\bullet}\right)
$$

из гомологической длинной точной последовательности пары $(W, W \backslash X)$ и изоморфизма

$$
H_{m}\left(W, W \backslash X ; \mathbf{L}_{\bullet}\right) \longrightarrow H_{m-q^{\prime}}\left(X ; \mathbf{L}_{\bullet}\right)
$$

Это аналогично ситуации в предложении 2. Вертикальные отображения в квадрате (32) индуцируют отображение между гомотопическими слоями горизонтальных отображений [14], и мы получаем гомотопически коммутативный квадpat

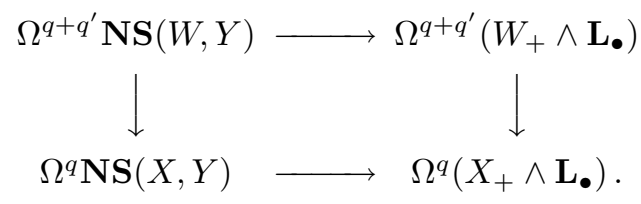

Он будет универсально-отталкивающим, поскольку кослои горизонтальных отображений естественно гомотопически эквивалентны спектру $\mathbf{L}\left(\pi_{1}(Y)\right)$. Коса точных последовательностей, определяемая (33), дана в диаграмме (31).

\section{Список литературы}

[1] A. A. Ranicki, Exact sequences in the algebraic theory of surgery, Math. Notes, 26, Princeton Univ. Press, Princeton, NJ; Univ. of Tokyo Press, Tokyo, 1981.

[2] A. A. Ranicki, "The total surgery obstruction", Algebraic topology (Proc. Sympos., Univ. Aarhus, Aarhus 1978), Lecture Notes in Math., 763, Springer-Verlag, Berlin, 1979, 275-316.

[3] A. Bak, "Odd dimension surgery groups of odd torsion groups vanish", Topology, 14:4 (1975), 367-374. 
[4] A. Bak, "The computation of surgery groups of finite groups with abelian 2hyperelementary subgroups", Algebraic K-theory (Proc. Conf., Northwestern Univ., Evanston, IL, 1976), Lecture Notes in Math., 551, Springer-Verlag, Berlin, 1976, 384409.

[5] I. Hambleton, I. Madsen, "On the computation of the projective surgery obstruction groups", K-theory, 7:6 (1993), 537-574.

[6] C. T. C. Wall, "Classification of Hermitian forms. VI: Group rings", Ann. of Math. (2), 103:1 (1976), 1-80.

[7] S. C. Ferry, A. A. Ranicki, J. Rosenberg (eds.), Novikov conjectures, index theorems and rigidity, vol. 1 (Oberwolfach, 1993), London Math. Soc. Lecture Note Ser., 226, Cambridge Univ. Press, Cambridge, 1995.

[8] A. A. Ranicki, Algebraic L-theory and topological manifolds, Cambridge Tracts in Math., 102, Cambridge Univ. Press, Cambridge, 1992.

[9] C. T. C. Wall, Surgery on compact manifolds, Second Edition, Math. Surveys Monogr., 69, ed. A. A. Ranicki, Amer. Math. Soc., Providence, RI, 1999.

[10] I. Hambleton, A. Ranicki, L. Taylor, "Round L-theory", J. Pure Appl. Algebra, 47:2 (1987), 131-154.

[11] Ю.В.Муранов, И. Хэмблтон, "Проективные группы препятствий к расщеплению вдоль односторонних подмногообразий", Матем. сб., 190:10 (1999), 65-86.

[12] Ю. В. Муранов, "Группы препятствий к расщеплению и квадратичные расширения антиструктур", Изв. РАН. Сер. матем., 59:6 (1995), 107-132.

[13] Ю. В. Муранов, Д. Реповш, "Группы препятствий к перестройкам и расщеплениям для пары многообразий", Матем. сб., 188:3 (1997), 127-142.

[14] R. Switzer, Algebraic topology-homotopy and homology, Grund. Math. Wiss., 212, Springer-Verlag, New York, 1975.

[15] S. López de Medrano, Involutions on manifolds, Ergeb. Math. Grezgeb., 59, SpringerVerlag, New York, 1971.

A. Бак (A. Bak)

University of Bielefeld, Germany

E-mail: bak@mathematik.uni-bielefeld.de

\section{Ю. В. Муранов (Yu. V. Muranov)}

Витебский государственный университет, Беларусь

E-mail: ymuranov@mail.ru
Поступила в редакцию

23.05 .2005 